\title{
Abnormal expression of TRIAP1 and its role in gestational diabetes mellitus-related pancreatic $\beta$ cells
}

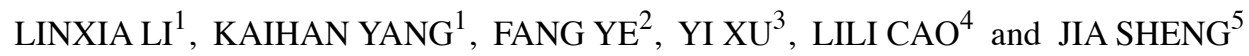 \\ Departments of ${ }^{1}$ Obstetrics and Gynecology, ${ }^{2}$ Biochemistry, ${ }^{3}$ Internal Medicine and \\ ${ }^{4}$ Microbiological Immunology, Chengdu University, Chengdu, Sichuan 610106; ${ }^{5}$ Department of Nursing, \\ Obstetrics and Gynecology, Hospital of Fudan University, Shanghai 200011, P.R. China
}

Received November 21, 2019; Accepted September 18, 2020

DOI: $10.3892 /$ etm.2021.9618

\begin{abstract}
Gestational diabetes mellitus (GDM) is a disease that is typically characterized by insulin resistance and pancreatic $\beta$ cell dysfunction. Currently, the role of TP53-regulated inhibitor of apoptosis 1 (TRIAP1) in the process of GDM remains to be elucidated. Therefore, the present study investigated the effects of TRIAP1 on GDM-related pancreatic $\beta$ cells. Reverse transcription-quantitative PCR and western blot assays were conducted to analyze the expression levels of TRIAP1 in the peripheral blood of patients with GDM and subjects with healthy pregnancies. Subsequently, TRIAP1 small interfering RNA (siRNA), control siRNA, TRIAP1 plasmid and control plasmid were transfected into INS-1 cells to assess the effects of TRIAP1 on pancreatic $\beta$ cells. ELISA was used to assess the total insulin content and insulin secretion of pancreatic $\beta$ cells. MTT and flow cytometry assays were performed to determine the viability and apoptosis of pancreatic $\beta$ cells. The results demonstrated that TRIAP1 expression was downregulated in peripheral blood samples from patients with GDM. Transfection with TRIAP1 siRNA significantly decreased the levels of total insulin content and reduced insulin secretion in pancreatic $\beta$ cells. In addition, downregulation of TRIAP1 in pancreatic $\beta$ cells significantly induced cell apoptosis and reduced cell viability. Accordingly, transfection of INS1 cells with TRIAP1 siRNA increased the levels of the apoptosis-associated genes apoptotic protease-activating factor 1 , caspase-3, caspase-7 and caspase-9. However, transfection of the cells with TRIAP1 plasmid resulted in the opposite effects. TRIAP1 increased the growth of pancreatic $\beta$ cells and their ability to secrete insulin, thus playing a protective role in GDM. The findings verified the effects and the underlying
\end{abstract}

Correspondence to: Mrs. Jia Sheng, Department of Nursing, Obstetrics and Gynecology, Hospital of Fudan University, 128 Shenyang Road, Shanghai 200011, P.R. China

E-mail: shengjia1114@163.com

Key words: gestational diabetes mellitus, TP53-regulated inhibitor of apoptosis 1 , pancreatic $\beta$ cells mechanism of TRIAP1 in pancreatic $\beta$ cells and may provide additional clinical applications for the therapy of GDM.

\section{Introduction}

Gestational diabetes mellitus (GDM) is a type of diabetes characterized by abnormal insulin deficiency and pancreatic $\beta$ cell dysfunction that occurs in pregnant females with a prevalence of $6-20 \%$ worldwide $(1,2)$. It is one of the most common diseases that occurs during pregnancy, normally leading to serious complications, such as cardiovascular illness, type 2 diabetes and obesity, as well as an increase in fetal morbidity and mortality $(3,4)$. During the process of GDM, insulin secretion is defective and pancreatic $\beta$ cells are unable to compensate for insulin resistance, inevitably leading to hyperglycemia (5). Recently, lifestyle habits, including diet and physical activity, have been suggested as key factors that can prevent the symptoms and development of GDM in females (6). However, in patients with GDM where lifestyle management cannot result in the recommended glycemia, additional therapeutic treatments, including exercise and insulin therapy, are required to control GDM $(7,8)$. Therefore, it is crucial to identify additional therapeutic strategies for GDM.

Dysfunction of pancreatic $\beta$-cells have been reported to be a central component in the pathogenesis and development of GDM $(9,10)$. Reduction of pancreatic $\beta$ cell function is characterized by increased rates of cell apoptosis (11) and defects in insulin generation and secretion (12). During pregnancy, insulin resistance results in increments of the insulin requirement (13), while insulin secretion by $\beta$ cells is reduced under the stimulation of prolactin and placental lactogens, and fetuin generation and secretion (12). During pregnancy, insulin resistance results in increments of the insulin requirement (13), while insulin secretion by $\beta$ cells is reduced under the stimulation of prolactin, placental lactogens and fetal growth (14-16). It was shown that hyperglycemia participated in the induction of pancreatic $\beta$ cell apoptosis (17) and insulin secretion (18). Therefore, investigating preventive mechanisms that can maintain glucose homeostasis by protecting and improving the functions of pancreatic $\beta$ cells is a significant research topic in the field of maternal medicine. However, to the best of our knowledge, the cellular and molecular mechanisms of pancreatic $\beta$ cell dysfunction remain to be elucidated. 
TP53-regulated inhibitor of apoptosis 1 (TRIAP1) is a small conserved protein containing 76 amino acids that was initially identified as a p53-induced cell survival factor $(19,20)$. It was previously reported that TRIAP1 results in cell death resistance via a mitochondrial-dependent pathway $(21,22)$. It was also shown that TRIAP1 can modulate apoptotic pathways by interacting with Hsp70-binding protein 1, prevent the formation of apoptotic protease-activating factor 1 (APAF1), release of cytochrome $c$ and induction of caspase-9, which reduced the formation of the apoptosome complex (23). These molecular events lead to insulin resistance by suppressing apoptosis and allowing DNA damage repair (23). Due to the significant function of TRIAP1 in apoptosis and the interaction between pancreatic $\beta$ cell apoptosis and GDM, the current study explored the involvement of TRIAP1 in the regulation of GDM.

\section{Materials and methods}

Clinical sample collection. Peripheral blood samples were collected from 30 female patients with GDM (age range, 24-37 years) and 30 female subjects that were undergoing healthy pregnancies (age range, 23-36 years) at the Obstetrics and Gynecology Hospital of Fudan University Hospital (Shanghai, China) between December 2017 and December 2018. The blood samples were separated by centrifugation $(1,000 \mathrm{x} \mathrm{g}$ for $10 \mathrm{~min}$ at $4^{\circ} \mathrm{C}$ ) and stored in liquid nitrogen instantly for subsequent experiments. There were no statistically significant differences in age, gestational week and weight between patients with GDM and subjects with healthy pregnancies. The present study was approved by the Ethics Committee of the Obstetrics and Gynecology Hospital of Fudan University, and written informed consent was acquired from all participants prior to their enrollment.

Females with a fasting plasma glucose (FPG) level of $\geq 4.4 \mathrm{mmol} / \mathrm{l}$ but $\leq 5.1 \mathrm{mmol} / 1$ underwent a $75 \mathrm{~g}$ oral glucose tolerance test (OGTT). In such cases, a diagnosis of GDM was made when at least one glucose value was elevated (FPG $\geq 5.1 \mathrm{mmol} / 1,1-\mathrm{h}$ OGTT $\geq 10.0 \mathrm{mmol} / 1$ or 2 -h OGTT $\geq 8.5 \mathrm{mmol} / \mathrm{l}$ ). Pregnant females with the following conditions were excluded from the present study: i) abnormal blood lipid (low-density lipoprotein cholesterol, triglyceride and high-density lipoprotein cholesterol) levels, hypertension and chronic kidney and liver diseases; ii) endocrine diseases, including obesity, diabetes, thyroid disease, osteoporosis, adrenal cortical disease and hyperthyroidism prior to pregnancy; iii) pregnant females currently undergoing long-term drug treatments (such as sodiumlevothyroxine) and iv) other pregnancy complications (pre-eclampsia, pregnancy-induced hypertension or pregnancy with chronic nephritis).

Cell culture and transfection. INS-1 cells were obtained from American Type Culture Collection. The cells were cultured and maintained in RPMI-1640 medium (HyClone; Cytiva) supplemented with $10 \%$ fetal bovine serum (HyClone; Cytiva), $11.1 \mathrm{mmol} / \mathrm{l}$ glucose, $50 \mathrm{mM}$ b-mercaptoethanol and $1 \%$ penicillin/streptomycin. The cells were maintained in $5 \% \mathrm{CO}_{2}$ at $37^{\circ} \mathrm{C}$.

TRIAP1-small interfering RNA (siRNA; $0.2 \mu \mathrm{M} ; 5$ '-AGG CAUGCACGGACAUGAATT-3') or control-siRNA $(0.2 \mu \mathrm{M}$;
5'-GCACCACGTGACGGAGCGT-3'), $1 \mu \mathrm{g}$ TRIAP1 CRISPR activation plasmid (cat. no. sc-427287-ACT) or control-plasmid (cat no. sc-437275) were purchased from Santa Cruz Biotechnology, Inc. Lipofectamine ${ }^{\circledR} 2000$ transfection reagent (Invitrogen; Thermo Fisher Scientific, Inc.) was used for transfection according to the manufacturer's instructions. Following transfection, the cells were cultured for $48 \mathrm{~h}$ and collected for further experiments. Cells without any treatment were used as the control.

Reverse transcription-quantitative PCR (RT-qPCR). Total RNA was isolated from peripheral blood and INS-1 cells using TRIzol $^{\circledR}$ reagent (Invitrogen; Thermo Fisher Scientific, Inc.). RNA concentration was detected using a NanoDrop ND-1000 spectrophotometer (Thermo Fisher Scientific, Inc.) at wavelengths of 260 and $180 \mathrm{~nm}$. Subsequently, $800 \mathrm{ng}$ RNA was reversed transcribed into cDNA using HiScriptTM Q RT SuperMix (Vazyme Biotech Co., Ltd.). The following temperature conditions for reverse transcription were as follows: $70^{\circ} \mathrm{C}$ for $5 \mathrm{~min}, 37^{\circ} \mathrm{C}$ for $5 \mathrm{~min}$ and $42^{\circ} \mathrm{C}$ for $60 \mathrm{~min}$. qPCR was performed using an ABI Prism 7500 Real-Time PCR System (Applied Biosystems; Thermo Fisher Scientific, Inc.) with the SYBR-Green Real-Time PCR kit (Toyobo Life Science) for the detection of RNA expression levels. The following thermocycling conditions were used for the qPCR: Initial denaturation for $5 \mathrm{~min}$ at $95^{\circ} \mathrm{C}$, followed by 35 cycles of denaturation at $94^{\circ} \mathrm{C}$ for $1 \mathrm{~min}$, annealing at $60^{\circ} \mathrm{C}$ for $1 \mathrm{~min}$ and extension at $72^{\circ} \mathrm{C}$ for $1 \mathrm{~min}$, followed by a final extension step at $72^{\circ} \mathrm{C}$ for $10 \mathrm{~min}$. GAPDH was used as the internal control and the relative expression levels of the transcripts were calculated using the $2^{-\Delta \Delta \mathrm{Cq}}$ method (24). Primer sequences were listed as following: TRIAP1 forward, 5'-GCACCGACCTCTTCA AGC-3' and reverse, 5'-CCATGAACTCCAGTCCTTCA3'; GAPDH forward, 5'-CTTTGGTATCGTGGAAGGACTC-3' and reverse, 5'-GTAGAGGCAGGGATGATGTTCT-3'; APAF1 forward, 5'-AACCAGGATGGGTCACCATA-3' and reverse, 5'-ACTGAAACCCAATGCACTCC-3'; caspase-3 forward, 5'-AGAACTGGACTGTGGCATTG-3' and reverse, 5'-CACAAAGCGACTGGATGAAC-3'; caspase-7 forward, 5'-CTACCGCCGTGGGAACGATGGCAGA-3' and reverse, 5'-CGAAGGCCCATACCTGTCACTTTATC-3' and caspase-9 forward, 5'-TTCCCAGGTTTTGTTTCCTG-3' and reverse, 5'-CCTTTCACCGAAACAGCATT-3'.

Western blot analysis. To obtain total protein, INS-1 cells and peripheral blood samples were lysed with RIPA buffer containing proteinase inhibitors (Beyotime Institute of Biotechnology) for $30 \mathrm{~min}$ and subsequently centrifuged at $12,000 \mathrm{x} \mathrm{g}$ for $3 \mathrm{~min}$ at $4^{\circ} \mathrm{C}$. Protein concentration was determined using a bicinchoninic acid protein assay kit (Beyotime Institute of Biotechnology). Subsequently, the proteins were mixed with $4 \mathrm{X}$ loading buffer, boiled at $95^{\circ} \mathrm{C}$ for $10 \mathrm{~min}$ and centrifuged at $10,000 \times \mathrm{g}$ at $4^{\circ} \mathrm{C}$ for $1 \mathrm{~min}$. Equal amounts of protein $(20 \mu \mathrm{g})$ were loaded on $10 \%$ SDS gels and analyzed by PAGE. The gels were transferred to PVDF membranes, and the membranes were blocked with $5 \%$ non-fat milk for $1 \mathrm{~h}$ at room temperature. The membranes were washed with PBS-0.1\% Tween-20 (PBST) three times and subsequently incubated overnight at $4^{\circ} \mathrm{C}$ with the following primary antibodies: TRIAP1 (cat. no. 515801; 1:1,000; Santa Cruz 
A

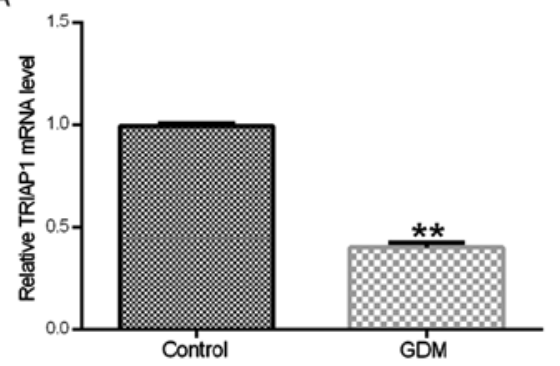

B

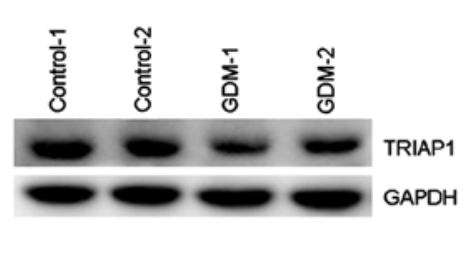

C

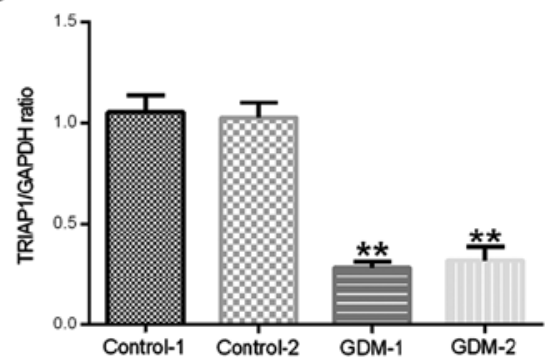

Figure 1. TRIAP1 exhibits low expression in patients with GDM. The expression levels of TRIAP1 in peripheral blood samples obtained from 30 patients with GDM and subjects with healthy pregnancies were detected by (A) reverse transcription-quantitative PCR and (B) western blotting. (C) The ratio of TRIAP1/GAPDH was calculated and presented. All experiments were performed in triplicate. The results are presented as the mean \pm standard deviation. ${ }^{* *} \mathrm{P}<0.01$ vs. control. GDM, gestational diabetes mellitus; TRIAP1, TP53-regulated inhibitor of apoptosis 1 .

Biotechnology, Inc.), APAF1 (cat. no. 8723; 1:1,000; Cell Signaling Technology, Inc.), caspase-3 (cat. no. 14220; 1:1000; Cell Signaling Technology, Inc.), caspase-7 (cat. no. 12827; 1:1,000; Cell Signaling Technology, Inc.), caspase-9 (cat. no. 9508; 1:1,000; Cell Signaling Technology, Inc.) and GAPDH (cat. no. 5174; 1:1,000; Cell Signaling Technology, Inc.). The following day, the membranes were washed with PBST four times and subsequently incubated with horseradish peroxidase-conjugated anti-rabbit immunoglobulin $\mathrm{G}$ secondary antibody (cat. no. 7074; 1:2,000; Cell Signaling Technology, Inc.) for $1 \mathrm{~h}$ at room temperature. Finally, protein bands were visualized using the ECL Western blot substrate (Pierce; Thermo Fisher Scientific, Inc.) and the relative band intensities were analyzed using ImageJ software version $1.48 \mathrm{u}$ (National Institutes of Health).

Cell viability assay. INS-1 cells were transfected with TRIAP1-siRNA, control-siRNA, TRIAP1-plasmid and control-plasmid, and then seeded into 96-well plates at a density of $1 \times 104$ cells/well. Following incubation for $48 \mathrm{~h}$ at $37^{\circ} \mathrm{C}$, $10 \mu \mathrm{l}$ MTT solution (5 mg/ml; Sigma-Aldrich; Merck KGaA) was added to each well and the cells were cultured for $4 \mathrm{~h}$ at $37^{\circ} \mathrm{C}$. Following incubation, DMSO (200 $\mu \mathrm{l}$; Sigma-Aldrich; Merck KGaA) was added to each well and the plate was shaken for $10 \mathrm{~min}$. Finally, the absorbance was measured using a Neo Multi-Mode Reader (BioTek Instruments, Inc.) at a wavelength of $570 \mathrm{~nm}$.

Flow cytometry analysis. To detect cell apoptosis, INS-1 cells were transfected with TRIAP1-siRNA, control-siRNA, TRIAP1-plasmid and control-plasmid, and then cultured for $48 \mathrm{~h}$. Following transfection, the Annexin V/PI Cell Apoptosis Detection kit (Nanjing KeyGen Biotech Co., Ltd) was used to determine the percentage of apoptotic cells. A total of $1 \times 10^{6}$ INS-1 cells were harvested and resuspended in $500 \mu 1$ binding buffer containing $5 \mu \mathrm{l}$ Annexin $\mathrm{V}$ and $5 \mu \mathrm{l}$ propidium iodide. The resulting solution was incubated for $15 \mathrm{~min}$ in the dark and the BD FACSCalibur ${ }^{\mathrm{TM}}$ flow cytometer (BD Biosciences) was used to evaluate the fluorescence intensity. Data were analyzed using the CellQuest ${ }^{\mathrm{TM}}$ version 5.1 software (BD Biosciences).

Insulin secretion detection. INS-1 cells were transfected with TRIAP1-siRNA, control-siRNA, TRIAP1-plasmid and control-plasmid, and then cultured for $48 \mathrm{~h}$. Following trans- fection, the cells were cultured with normal glucose $(3.3 \mathrm{mM})$ or high glucose $(16.7 \mathrm{mM})$ for $1 \mathrm{~h}$. Subsequently, the total insulin content was extracted following sonication of the cells in acid ethanol $\left(2 \% \mathrm{H}_{2} \mathrm{SO}_{4}\right.$ in $75 \%$ ethanol), accompanied with three cycles of freezing and thawing. The cells were centrifuged at $500 \mathrm{x} \mathrm{g}$ at $4^{\circ} \mathrm{C}$ for $5 \mathrm{~min}$ and the supernatant was used to determine insulin release levels using ELISA kit (cat. no. PI606; Beyotime Institute of Biotechnology) according to the manufacturer's instructions.

Statistical analysis. Statistical analyses were performed with SPSS 21.0 software (IBM Corp.). The derived values were expressed as the mean \pm SD. One-way ANOVA with Tukey's post hoc test was used for comparisons between multiple groups. Unpaired Student's t-test was used to analyze the difference between two groups. $\mathrm{P}<0.05$ was considered to indicate a statistically significant difference.

\section{Results}

TRIAPI expression is downregulated in peripheral blood samples from patients with GDM. To examine the pathological relevance of TRIAP1 in GDM, the expression levels of TRIAP1 was detected in peripheral blood samples obtained from 30 patients with GDM and subjects with healthy pregnancies by RT-qPCR and western blot assays. As shown in Fig. 1A-C, the mRNA and protein expression levels of TRIAP1 in peripheral blood samples from patients with GDM were significantly lower compared with samples from healthy pregnancies.

Downregulation of TRIAPI suppresses insulin secretion and total insulin content. To determine whether TRIAP1 is involved in regulating pancreatic $\beta$ cell function, control-siRNA and TRIAP1-siRNA were transfected into INS-1 cells and the transfection efficiency was confirmed by RT-qPCR and western blot assays. As shown in Fig. 2A and B, transfection of cells with TRIAP1-siRNA significantly downregulated the mRNA and protein expression levels of TRIAP1 compared with the control-siRNA group. In addition, the results presented in Fig. $2 \mathrm{C}$ demonstrated that downregulation of TRIAP1 could inhibit the total insulin content in INS-1 cells compared with control-siRNA group. It has been frequently reported that high glucose can promote insulin secretion (25). 

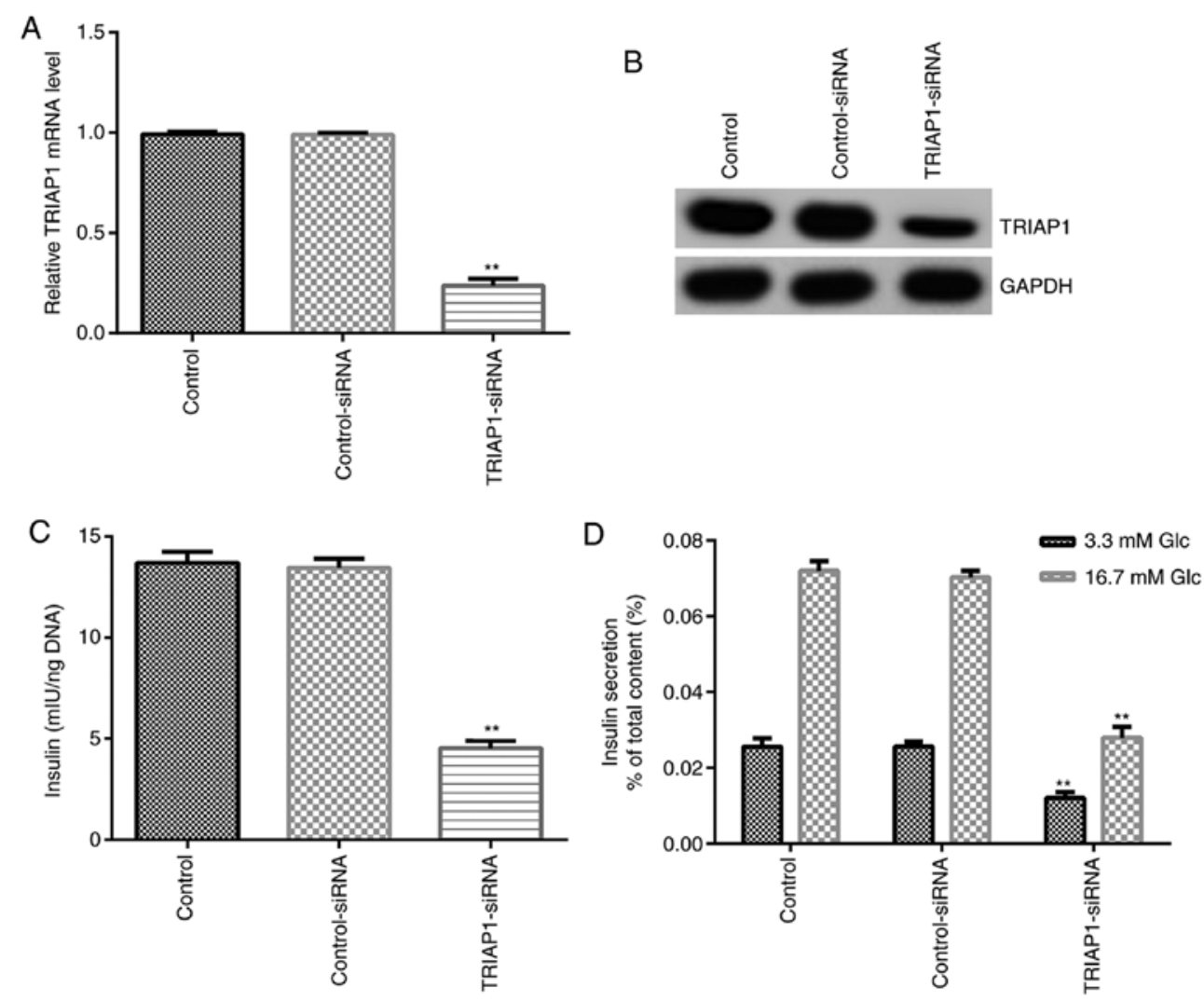

Figure 2. TRIAP1-siRNA inhibits insulin secretion and total insulin content. TRIAP1-siRNA and control-siRNA were transfected into INS-1 cells that were pretreated with 3.3 or $16.7 \mathrm{mM}$ glucose. The transfection efficiency was detected by (A) reverse transcription-quantitative PCR and (B) western blot assays. (C) The total insulin content and (D) insulin secretion were detected by ELISA in INS-1 cells. The experiments were performed in triplicate. The results are presented as the mean \pm standard deviation. ${ }^{* *} \mathrm{P}<0.01$ vs. control-siRNA. TRIAP1, TP53-regulated inhibitor of apoptosis 1 ; siRNA, small interfering RNA; Glc, glucose.

In the present study, the data showed that downregulation of TRIAP1 significantly inhibited insulin release under high and low glucose conditions in INS-1 cells compared with control-siRNA groups (Fig. 2D). The aforementioned results indicated that downregulation of TRIAP1 expression suppressed total insulin content and reduced insulin release in INS-1 cells under glucose stimulation.

Downregulation of TRIAPI effects INS-1 cell viability and apoptosis. Subsequently, the effects of TRIAP1 on the viability and apoptosis of INS-1 cells were investigated. Control-siRNA and TRIAP1-siRNA were transfected into INS- 1 cells for $48 \mathrm{~h}$, and MTT and flow cytometry analyses were performed to detect the viability and apoptosis of INS-1 cells, respectively. As shown in Fig. 3A-C, downregulation of TRIAP1 significantly decreased cell viability and significantly increased the cell apoptotic population compared with their respective control-siRNA groups. Furthermore, the expression levels of cell apoptosis-associated genes, including APAF1, caspase- 3 , caspase- 7 and caspase- 9 , were determined by RT-qPCR and western blotting. The findings demonstrated that the protein and mRNA expression levels of APAF1, caspase-3, caspase-7 and caspase- 9 were significantly increased in the TRIAP1-siRNA-transfected group compared with the control-siRNA group (Fig. 3D-H). The aforementioned results indicated that TRIAP1-siRNA suppressed insulin secretion by inhibiting cell viability, promoting cell apoptosis and regu- lating the expression of apoptosis-associated genes in INS-1 cells.

Upregulation of TRIAP1 promotes insulin secretion and total insulin content. Subsequently, the regulation of TRIAP1 expression was investigated with regard to the metabolic function of pancreatic $\beta$ cells. INS-1 cells were transfected with TRIAP1-plasmid and control-plasmid and the transfection efficiency was evaluated by RT-qPCR and western blot assays. As shown in Fig. 4A and B, the mRNA and protein expression levels of TRIAP1 were significantly increased in the TRIAP1-plasmid group compared with the control-plasmid group. The results also demonstrated that upregulation of TRIAP1 expression could significantly increase the total insulin content in INS-1 cells compared with control-plasmid group (Fig. 4C). Upregulation of TRIAP1 expression further significantly promoted insulin secretion under high and low glucose conditions in INS- 1 cells compared with the control-plasmid group (Fig. 4D). These data suggest that TRIAP1 serves an essential role in regulating insulin secretion of pancreatic $\beta$ cells.

Upregulation of TRIAPI influences of INS-1 cell viability and apoptosis. To further investigate the role of TRIAP1 in the regulation of proliferation and apoptosis of INS-1 cells, control-plasmid and TRIAP1-plasmids were transfected into the cells for $48 \mathrm{~h}$. MTT assay and flow cytometry analysis 


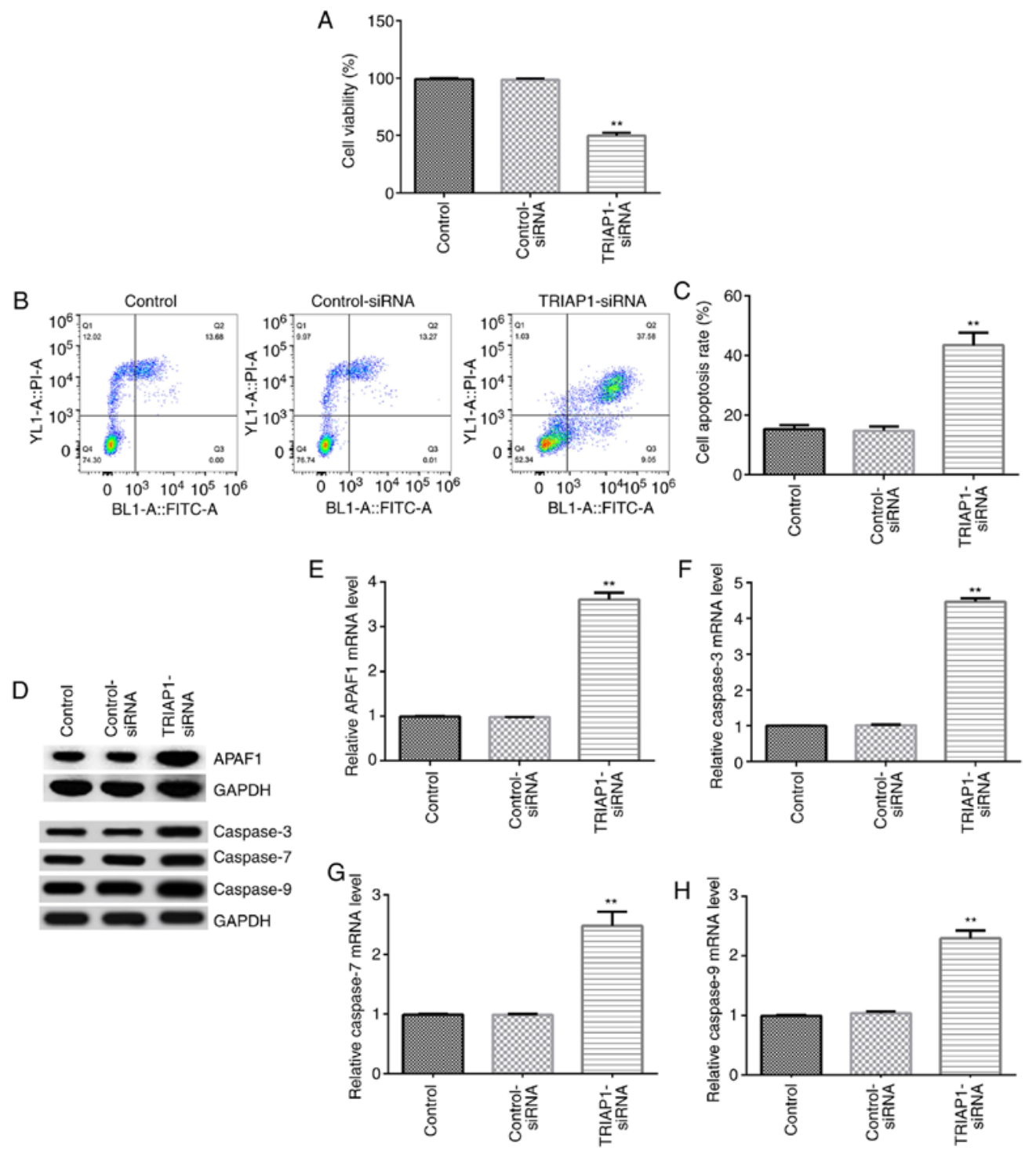

Figure 3. Effects of TRIAP1-siRNA on INS-1 cell viability and apoptosis. TRIAP1-siRNA and control-siRNA were transfected into INS-1 cells for $48 \mathrm{~h}$. Cell viability and apoptosis of INS-1 cells were evaluated by (A) MTT and (B and C) flow cytometry assays. (D) Western blot showing the expression levels of apoptosis-associated genes. reverse transcription-quantitative PCR results showing the expression levels of apoptosis-associated genes (E) APAF1, (F) caspase-3, (G) caspase-7 and (H) caspase-9. Note: The blots of APAF1 and its internal control GAPDH were from the different parts of the same gel; and the blots of caspase-3, caspase-7 and caspase-9 and the internal control GAPDH were from the different parts of the same gel. All experiments were conducted in triplicate. The results are presented as the mean \pm standard deviation. ${ }^{* *} \mathrm{P}<0.01$ vs. control-siRNA. TRIAP1, TP53-regulated inhibitor of apoptosis 1 ; siRNA, small interfering RNA; APAF1, apoptotic protease-activating factor 1.

indicated that upregulation of TRIAP1 significantly increased cell viability and significantly decreased apoptosis of INS-1 cells (Fig. 5A-C) compared with the control-plasmid groups. Western blotting and RT-qPCR results demonstrated that the protein and mRNA expression levels of APAF1, caspase-3, caspase-7 and caspase- 9 were significantly decreased in the TRIAP1-plasmid-transfected group compared with the control-plasmid group (Fig. 5D-H). Taken together, these findings demonstrated that TRIAP1 could influence insulin secretion by regulating cell viability, cell apoptosis and the expression levels of apoptosis-associated genes in INS-1 cells.

\section{Discussion}

GDM is a common condition in which hyperglycemia occurs in females during pregnancy (26), which usually leads to an enhanced risk of adverse outcomes for mothers and newborns (27). It was reported that during pregnancy, strict control of blood glucose can ameliorate potential poor health outcomes of the mothers and newborns during pregnancy (28). Individualized management, including exercise, dietary guidance and encouragement for physical activity, was suggested to improve glycemic control in patients with GDM (29). When lifestyle management fails to retain adequate glycemic levels, alternative therapeutic strategies, such as exercise and insulin therapy, may be used for GDM $(7,8)$. Recently, a study demonstrated that pancreatic $\beta$ cell dysfunction plays an important role in the pathogenesis of GDM (30). TRIAP1 is a small conserved protein containing 76 amino acids that is considered a regulator of cell death $(19,20)$. However, the role of TRIAP1 in pancreatic $\beta$ cells remains to be investigated. Therefore, the aim of the present study was to assess the regulatory effects of 

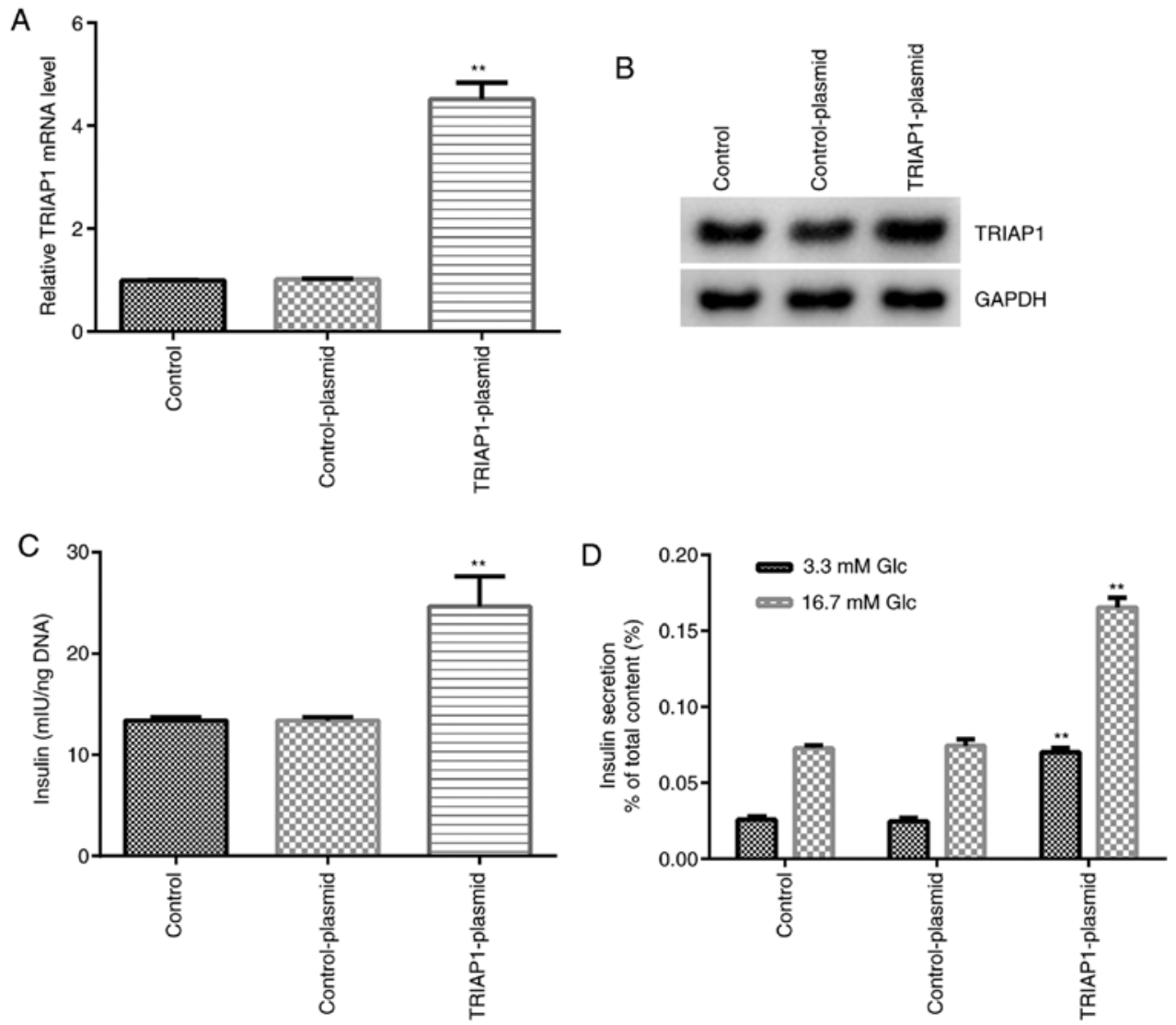

Figure 4. TRIAP1 overexpression promotes insulin secretion and increases total insulin content. TRIAP1-plasmid and control-plasmid were transfected into INS-1 cells that were pretreated with 3.3 or $16.7 \mathrm{mM}$ glucose. (A) Reverse transcription-quantitative PCR and (B) western blot assays were performed to determine transfection efficiency. (C) Total insulin content and (D) insulin secretion were detected using ELISA in INS-1 cells. The experiments were conducted in triplicate. The results are presented as the mean \pm standard deviation. ${ }^{* *} \mathrm{P}<0.01$ vs. control-plasmid. TRIAP1, TP53-regulated inhibitor of apoptosis 1 ; Glc, glucose.

TRIAP1 in GDM and its functions in pancreatic $\beta$ cells. It was hypothesized that TRIAP1 played critical roles in regulating pancreatic $\beta$ cell function.

In the present study, RT-qPCR and western blot assays were used to determine the levels of TRIAP1 in GDM, and the data revealed that TRIAP1 expression was downregulated in peripheral blood samples from patients with GDM compared with those obtained from control subjects. Since the present study focused on the investigation of TRIAP1 in GDM, a control group for patients with diabetes was not included in the current study (31). However, the number of samples of the present study is too small, and large sample needs to be included. Additionally, the association between clinicopathological characteristics of patients with GDM and TRIAP1 expression was not analyzed in the present study. These were the limitations of the present study and will be further studied in the future. To further investigate the effects of TRIAP1 in GDM, TRIAP1-siRNA and control-siRNA were transfected into INS-1 cells. The transfection efficiency was confirmed by RT-qPCR and western blot assays, and the results indicated that TRIAP1-siRNA significantly downregulated the mRNA and protein expression levels of TRIAP1. Pancreatic $\beta$ cells are a distinct population of cells that produce insulin in order to fulfill metabolic demands (32). The lack of adaptation of pancreatic $\beta$ cells to peripheral insulin resistance may be the main cause of GDM $(33,34)$. Therefore, ELISA was performed to evaluate insulin secretion and total insulin content in INS-1 cells under high and low glucose conditions. ELISA results revealed that TRIAP1-siRNA significantly reduced the total insulin content and insulin release in INS-1 cells. However, further confirm the results, observation of changes in cell and insulin secretion by setting different time gradients and concentration gradients in cell experiments is required, which will be investigated this in the future.

A previous study demonstrated that inhibition of TRIAP1 in RPMI8226 cells increased the proportion of apoptotic cells, increased the expression levels of APAF1 and caspase-9, and induced caspase- 9 and caspase-3/7 activities (35). Therefore, the effects of TRIAP1 knockdown on the proliferation and apoptosis of INS-1 cells was investigated in the present study. In line with previous studies $(19,35,36)$, the results indicated that TRIAP1-siRNA may lead to the induction of apoptosis and to the inhibition of INS-1 cell proliferation. The data revealed that TRIAP1-siRNA promoted apoptosis of pancreatic $\beta$ cells. Furthermore, the results from the RT-qPCR and western blot assays demonstrated that TRIAP1-siRNA significantly increased the expression levels of the apoptosis-associated genes APAF1, caspase-3, caspase-7 and caspase9 in INS-1 cells.

Accordingly, TRIAP1 overexpression enhanced insulin secretion and total insulin content in INS-1 cells, as demonstrated by transfection of the cells with TRIAP1-plasmid. In 

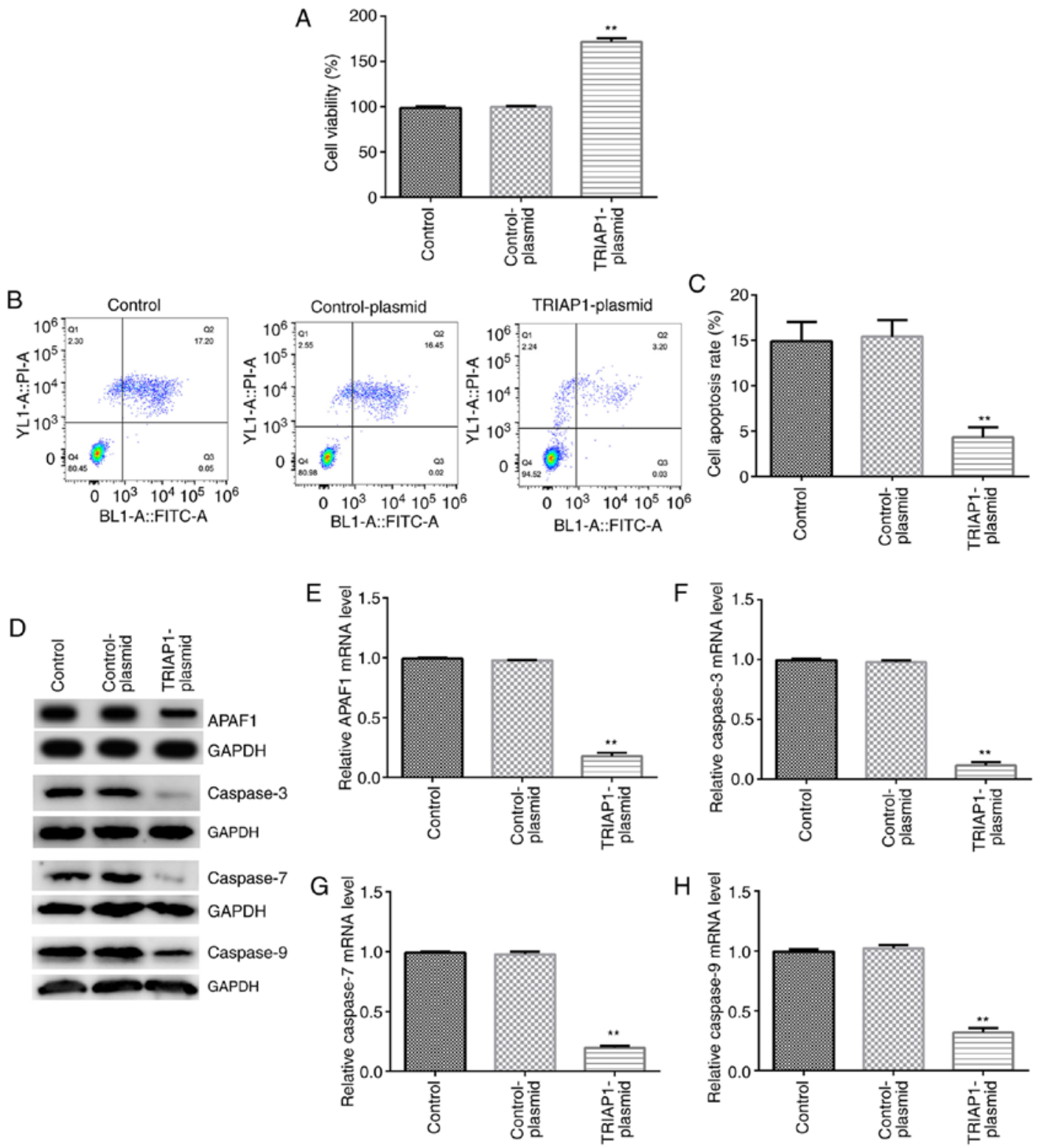

Figure 5. Effects of TRIAP1-plasmid on INS-1 cell viability and apoptosis. TRIAP1-plasmid and control-plasmid were transfected into INS-1 cells for $48 \mathrm{~h}$. (A) MTT and (B and C) flow cytometry assays were performed to analyze the viability and apoptosis of INS-1 cells. (D) Western blot showing the expression levels of apoptosis-associated genes. Reverse transcription-quantitative PCR results showing the expression levels of apoptosis-associated genes (E) APAF1, (F) caspase-3, (G) caspase-7 and (H) caspase-9. Note: The blots of APAF1 and its internal control GAPDH were from the different parts of the same gel; the blots of caspase-3 and the internal control GAPDH were from the different parts of the same gel; the blots of caspase-7 and the internal control GAPDH were from the different parts of the same gel; and the blots of caspase- 9 and the internal control GAPDH were from the different parts of the same gel. All experiments were conducted in triplicate. The results are presented as the mean \pm standard deviation. ${ }^{* * *} \mathrm{P}<0.01$ vs. control-plasmid. TRIAP1, TP53-regulated inhibitor of apoptosis 1; APAF1, apoptotic protease-activating factor 1.

addition, TRIAP1 overexpression significantly inhibited the induction of INS-1 cell apoptosis and promoted the viability of INS-1 cells. Moreover, the results demonstrated the repressive effects of TRIAP1 overexpression on the expression levels of APAF1, caspase-3, caspase-7 and caspase-9 in INS-1 cells . The aforementioned findings demonstrated that TRIAP1 expression was downregulated in patients with GDM and that it may play an important role in pancreatic $\beta$ cell function by regulating insulin secretion, as well as proliferation and apoptosis of pancreatic $\beta$ cells.

Taken together, the present study revealed a novel role of TRIAP1 in GDM. This protein may serve as a potential target for the therapy of GDM. The present study revealed that
TRIAP1 could regulate insulin secretion and the proliferation and apoptosis of pancreatic $\beta$ cells. This indicated a protective role of TRIAP1 in GDM and suggested novel possibilities for the use of targeted therapy against this disease. Although previous studies have analyzed the predictive and diagnostic biomarkers for GDM $(37,38)$, however, whether TRIAP1 plays a prognostic or diagnostic role in GDM needs further research, which will be performed in the future.

In conclusion, TRIAP1 increased the growth of pancreatic $\beta$ cells and their ability to secrete insulin, which in turn resulted in a protective effect in GDM. Therefore, TRIAP1 may be considered a promising therapeutic target for GDM treatment. 


\section{Acknowledgements}

Not applicable.

\section{Funding}

No funding was received.

\section{Availability of data and materials}

The datasets used and/or analyzed during the current study are available from the corresponding author on reasonable request.

\section{Authors' contributions}

LXL contributed to the conception and design of the study, data acquisition, analysis and interpretation. KHY, FY, YX and LLC contributed to data acquisition and performed statistical analysis. JS contributed to data acquisition, data analysis and prepared the manuscript. All authors read and approved the final manuscript.

\section{Ethics approval and consent to participate}

The present study was approved by the Ethics Committee of the Obstetrics and Gynecology Hospital of Fudan University (Shanghai, China) and written informed consent was acquired from all participants prior to their enrollment.

\section{Patient consent for publication}

Not applicable.

\section{Competing interests}

The authors declare that they have no competing interests.

\section{References}

1. Zhen XM, Li X and Chen C: Longer-term outcomes in offspring of GDM mothers treated with metformin versus insulin. Diabetes Res Clin Pract 144: 82-92, 2018.

2. Shan Z, Xu C, Wang W and Li W: Enhanced PDGF signaling in gestational diabetes mellitus is involved in pancreatic $\beta$-cell dysfunction. Biochem Biophys Res Commun 516: 402-407, 2019.

3. Goueslard K, Cottenet J, Mariet AS, Giroud M, Cottin Y, Petit JM and Quantin C: Early cardiovascular events in women with a history of gestational diabetes mellitus. Cardiovasc Diabetol 15: 15, 2016.

4. Gunderson EP, Hurston SR, Dewey KG, Faith MS, CharvatAguilar N, Khoury VC, Nguyen VT and Quesenberry CP Jr: The study of women, infant feeding and type 2 diabetes after GDM pregnancy and growth of their offspring (SWIFT Offspring study): prospective design, methodology and baseline characteristics. BMC Pregnancy Childbirth 15: 150, 2015.

5. Wolpin BM, Bao Y, Qian ZR, Wu C, Kraft P, Ogino S, Stampfer MJ, Sato K, Ma J, Buring JE, et al: Hyperglycemia, insulin resistance, impaired pancreatic $\beta$-cell function, and risk of pancreatic cancer. J Natl Cancer Inst 105: 1027-1035, 2013.

6. Malek R and Davis SN: Pharmacokinetics, efficacy and safety of glyburide for treatment of gestational diabetes mellitus. Expert Opin Drug Metab Toxicol 12: 691-699, 2016.

7. Aberg AEB, Jönsson EK, Eskilsson I, Landin-Olsson M and Frid AH: Predictive factors of developing diabetes mellitus in women with gestational diabetes. Acta Obstet Gynecol Scand 81: $11-16,2002$.

8. Alfadhli EM: Gestational diabetes mellitus. Saudi Med J 36: 399-406, 2015.
9. Plows JF, Stanley JL, Baker PN, Reynolds CM and Vickers MH: The Pathophysiology of Gestational Diabetes Mellitus. Int J Mol Sci 19: 3342, 2018.

10. Li L, Wang S, Li H, Wan J, Zhou Q, Zhou Y and Zhang C: microRNA-96 protects pancreatic $\beta$-cell function by targeting PAK1 in gestational diabetes mellitus. Biofactors 44: 539-547, 2018.

11. Chen C, Luo Y, Su Y and Teng L: The vitamin D receptor (VDR) protects pancreatic beta cells against Forkhead box class O1 (FOXO1)-induced mitochondrial dysfunction and cell apoptosis. Biomed Pharmacother 117: 109170, 2019.

12. Mandelbaum AD, Kredo-Russo S, Aronowitz D, Myers N, Yanowski E, Klochendler A, Swisa A, Dor Y and Hornstein E: miR-17-92 and miR-106b-25 clusters regulate beta cell mitotic checkpoint and insulin secretion in mice. Diabetologia 62: 1653-1666, 2019.

13. Vejrazkova D, Vcelak J, Vankova M, Lukasova P, Bradnova O, Halkova T, Kancheva R and Bendlova B: Steroids and insulin resistance in pregnancy. J Steroid Biochem Mol Biol 139: 122-129, 2014.

14. Hill DJ: Placental control of metabolic adaptations in the mother for an optimal pregnancy outcome. What goes wrong in gestational diabetes? Placenta 69: 162-168, 2018.

15. Brelje TC, Bhagroo NV, Stout LE and Sorenson RL: Beneficial effects of lipids and prolactin on insulin secretion and beta-cell proliferation: A role for lipids in the adaptation of islets to pregnancy. J Endocrinol 197: 265-276, 2008.

16. Boehmer BH, Brown LD, Wesolowski SR, Hay WW Jr and Rozance PJ: Pulsatile hyperglycemia increases insulin secretion but not pancreatic $\beta$-cell mass in intrauterine growth-restricted fetal sheep. J Dev Orig Health Dis 9: 492-499, 2018.

17. Weng Q, Zhao M, Zheng J, Yang L, Xu Z, Zhang Z, Wang J, Wang J, Yang B, Richard Lu Q, et al: STAT3 dictates $\beta$-cell apoptosis by modulating PTEN in streptozocin-induced hyperglycemia. Cell Death Differ 27: 130-145, 2020.

18. Li T, Ni L, Zhao Z, Liu X, Lai Z, Di X, Xie Z, Song X, Wang X, Zhang $\mathrm{R}$, et al: Melatonin attenuates smoking-induced hyperglycemia via preserving insulin secretion and hepatic glycogen synthesis in rats. J Pineal Res 64: e12475, 2018.

19. Adams C, Cazzanelli G, Rasul S, Hitchinson B, Hu Y, Coombes RC, Raguz S and Yagüe E: Apoptosis inhibitor TRIAP1 is a novel effector of drug resistance. Oncol Rep 34: 415-422, 2015.

20. Ketteler J, Panic A, Reis H, Wittka A, Maier P, Herskind C, Yagüe E, Jendrossek V and Klein D: Progression-related loss of stromal caveolin 1 levels mediates radiation resistance in prostate carcinoma via the apoptosis inhibitor TRIAP1. J Clin Med 8: 8, 2019

21. Li Y, Tang X, He Q, Yang X, Ren X, Wen X, Zhang J, Wang Y, Liu N and Ma J: Overexpression of mitochondria mediator gene TRIAP1 by miR-320b loss is associated with progression in nasopharyngeal carcinoma. PLoS Genet 12: e1006183, 2016.

22. Miliara X, Garnett JA, Tatsuta T, Abid Ali F, Baldie H, Pérez-Dorado I, Simpson P, Yague E, Langer T and Matthews S: Structural insight into the TRIAP1/PRELI-like domain family of mitochondrial phospholipid transfer complexes. EMBO Rep 16: 824-835, 2015.

23. Alyes VLF, Zanatta DB, de Oliveira MB, Eugenio AIP, Fernando RC, Strauss BE and Colleoni GWB: Silencing of apoptosome regulating genes, HSP70 and TRIAP1, induces apoptosis in MM cell lines. In: Proceedings of the 106th Annual Meeting of the American Association for Cancer Research, Philadelphia, PA, 2015. Cancer Res 75 (Suppl 15): Abstract nr 1017, 2015.

24. Livak KJ and Schmittgen TD: Analysis of relative gene expression data using real-time quantitative PCR and the 2(-Delta Delta C(T)) method. Methods 25: 402-408, 2001.

25. Zhong B, Ma S and Wang DH: TRPV1 mediates glucose-induced insulin secretion through releasing neuropeptides. In Vivo 33: 1431-1437, 2019.

26. Seshiah V, Kalra S, Gupte S, Divakar H, Murugananthan A, Banerjee S, Gupta S, Balaji V,Zargar A, Das A, et al: Classification of hyperglycemia in pregnancy. Indian J Endocrinol Metab 18: 445-448, 2014.

27. Dessì A, Marincola FC and Fanos V: Metabolomics and the great obstetrical syndromes - GDM, PET, and IUGR. Best Pract Res Clin Obstet Gynaecol 29: 156-164, 2015.

28. Naseh A, Ashrafzadeh S and Rassi S: Prevalence of vitamin D deficiency in pregnant mothers in Tehran and investigating its association with serum glucose and insulin. J Matern Fetal Neonatal Med 31: 2312-2318, 2018. 
29. Wang C, Zhu WW, Wei YM, Feng H, Su R and Yang HX: Exercise intervention during pregnancy can be used to manage weight gain and improve pregnancy outcomes in women with gestational diabetes mellitus. BMC Pregnancy Childbirth 15: $255,2015$.

30. Xu K, Bian D, Hao L, Huang F, Xu M, Qin J and Liu Y: microRNA-503 contribute to pancreatic beta cell dysfunction by targeting the mTOR pathway in gestational diabetes mellitus. EXCLI J 16: 1177-1187, 2017.

31. Zhang YL and Chen XQ: Dysregulation of microRNA-770-5p influences pancreatic- $\beta$-cell function by targeting TP53 regulated inhibitor of apoptosis 1 in gestational diabetes mellitus. Eur Rev Med Pharmacol Sci 24: 793-801, 2020.

32. Boland BB, Rhodes CJ and Grimsby JS: The dynamic plasticity of insulin production in $\beta$-cells. Mol Metab 6: 958-973, 2017.

33. Jian X and Felsenfeld G: Insulin promoter in human pancreatic $\beta$ cells contacts diabetes susceptibility loci and regulates genes affecting insulin metabolism. Proc Natl Acad Sci USA 115: E4633-E4641, 2018.

34. Miyakoshi K, Tanaka M, Saisho Y, Shimada A, Minegishi K, Kim SH, Asai S, Itoh $\mathrm{H}$ and Yoshimura Y: Pancreatic beta-cell function and fetal growth in gestational impaired glucose tolerance. Acta Obstet Gynecol Scand 89: 769-775, 2010.
35. Fook-Alves VL, de Oliveira MB, Zanatta DB, Strauss BE and Colleoni GWB: TP53 regulated inhibitor of apoptosis 1 (TRIAP1) stable silencing increases late apoptosis by upregulation of caspase 9 and APAF1 in RPMI8226 multiple myeloma cell line. Biochim Biophys Acta 1862: 1105-1110, 2016.

36. Potting C, Tatsuta T, König T, Haag M, Wai T, Aaltonen MJ and Langer T: TRIAP1/PRELI complexes prevent apoptosis by mediating intramitochondrial transport of phosphatidic acid. Cell Metab 18: 287-295, 2013.

37. Lorenzo-Almorós A, Hang T, Peiró C, Soriano-Guillén L, Egido J, Tuñón $\mathrm{J}$ and Lorenzo Ó: Predictive and diagnostic biomarkers for gestational diabetes and its associated metabolic and cardiovascular diseases. Cardiovasc Diabetol 18: 140, 2019.

38. Wójcik M, Mac-Marcjanek K, Woźniak LA, Nadel I, Lewiński A and Cypryk K: The association of leukocyte phosphatidylinositol 3-kinase delta overexpression with gestational diabetes mellitus (GDM). Endokrynol Pol 65: 17-24, 2014.

(c) (i) () () This work is licensed under a Creative Commons Attribution-NonCommercial-NoDerivatives 4.0 International (CC BY-NC-ND 4.0) License. 